\title{
A mysterious case with abdominal pain and syndrome of inappropriate anti-diuretic hormone secretion
}

\author{
Özlem Yüksel Aksoy ${ }^{1 \oplus}$, Mehmet Gündüz ${ }^{2 \oplus}$, Özlem Ünal ${ }^{2 \oplus}$, Funda Bostanc1 ${ }^{3 \oplus}$, \\ Fatma Şemsa Çayc1 ${ }^{1 \oplus}$, Umut Selda Bayrakc1 ${ }^{1 \oplus}$ \\ Divisions of ${ }^{1}$ Pediatric Nephrology and ${ }^{2}$ Pediatric Metabolism and Nutrition, ${ }^{3}$ Department of Pediatrics, University of Health Sciences, \\ Ankara Child Health and Diseases Hematology Oncology Training and Research Hospital, Ankara, Turkey.
}

\begin{abstract}
Background. Acute intermittent porphyria (AIP) is a rare, hereditary, metabolic disease caused by a defect in heme biosynthesis. Hormonal changes may trigger porphyria attacks.

Case. Here we present a 17 -year- old adolescent refugee mother who applied to the pediatric emergency department with the complaint of diffuse abdominal pain at puerperium. The patient was hypertensive, and had convulsions after admission. Hyponatremia (serum sodium; $121 \mathrm{meq} / \mathrm{L}$ ) was detected, and syndrome of inappropriate anti-diuretic hormone secretion (SIADH) was found to be the cause of hyponatremia which responded well to fluid restriction. Infectious, autoimmune and toxicologic laboratory work-up did not reveal any specific pathologies. Despite prompt utilization of analgesic treatment, the patient continued to have unbearable abdominal pain. The preference of prone position to relieve the pain and the family history of a mother who had died with similar symptoms, led us to the diagnosis of AIP. Genetic analysis showed a heterozygous mutation in hydroxymethylbilane synthase (HMBS) gene (c160+6T>A) which confirmed our diagnosis.
\end{abstract}

Conclusion. Acute porphyrias should be considered in differential diagnosis of abdominal pain, especially when there are accompanying symptoms like hyponatremia, seizures, mental changes and hypertension.

Key words: abdominal pain, acute intermittent porphyria, SIADH.

Acute porphyias are rare, metabolic diseases caused by the mutations of the genes involved in heme biosynthesis. ${ }^{1}$ Acute intermittent porphyria (AIP) is inherited in an autosomal dominant fashion and is the most common acute porphyria type in Europe. ${ }^{2}$ Certain drugs including oral contraceptives, hormonal changes, low carbohydrate intake are known precipitating factors for acute attacks. Vomiting and constipation accompanying abdominal pain may mimic acute abdomen in children. ${ }^{3}$ Electrolyte imbalances, especially hyponatremia

○ Özlem Yüksel Aksoy

ozlem_yurtsever@yahoo.com

Received 22nd January 2019, revised 27th May 2019, accepted 2nd January 2020.

The case has been presented as a written poster in 51st Annual ESPN Meeting, 5th October 2018, Antalya, Turkey. due to syndrome of inappropriate anti-diuretic hormone secretion (SIADH), may be seen during an attack.

Here, we present an adolescent refugee mother who was diagnosed with AIP. The porphyria attack manifested with abdominal pain, SIADH and hypertension at puerperium period.

\section{Case Report}

A seventeen -year- old Syrian refugee female patient applied to the pediatric emergency department with the complaints of vomiting and abdominal pain. The patient had given birth to her second child 35 days ago and she had constipation for more than a week. On physical examination, she was pale and looked like she was in pain. She had diffuse tenderness 
in her abdomen. Her blood pressure was 150/90 mmhg; the patient also had a tonic-clonic seizure when she was under observation.

In laboratory evaluation her serum sodium level was $121 \mathrm{meq} / \mathrm{L}$; hypertonic saline infusion was administered due to the seizure. Her serum potassium level was $4.4 \mathrm{meq} / \mathrm{L}$, chloride level was $83 \mathrm{meq} / \mathrm{L}$, serum urea was $58 \mathrm{mg} / \mathrm{dl}$, serum creatinine was $0.86 \mathrm{mg} / \mathrm{dl}$ and uric acid level was $7 \mathrm{mg} / \mathrm{dl}$. Serum glucose level and complete blood count were normal. In urinary dipstick test microscopic hematuria and positive urobilinogen were detected. Abdominal ultrasonography did not reveal any specific pathologies.

The patient was hospitalized for further investigation. Oral amlodipine and enalapril treatments were started in a stepwise manner for hypertension. Cranial imaging was normal. Hypophyseal hormone levels and pituitary magnetic resonance imaging showed normal findings, therefore Sheehan syndrome due to post-partum hemorrhage was excluded. Blood osmolarity, urinary osmolarity were $278 \mathrm{mOsm} /$ $\mathrm{kg}$ and $489 \mathrm{mOsm} / \mathrm{kg}$, respectively. Urinary sodium concentration was $>40 \mathrm{mEq} / \mathrm{L}$ and the patient was euvolemic. Volume restriction and salt replacement were performed with the diagnosis of SIADH. Despite normalization of serum sodium level, her severe abdominal pain persisted.

Amylase and lipase levels which were in normal range on admission (98 U/L and 4 $\mathrm{U} / \mathrm{L}$, respectively), rose on the tenth day of hospitalization(155U/Land41U/L, respectively). Repeated ultrasonographic evaluation was consistent with pancreatitis. However, magnetic resonance cholangiopancreatography was found to be normal except gallbladder sludge. Intravenous sulperazone treatment was started. During her follow-up, a decrease was observed in levels of amylase and lipase. Her abdominal pain had subsided as well. Due to the difficulties in communication and probably because of the postpartum depression, the follow-up was getting harder. We learned that the patient's mother had died at a young age with similar symptoms. During the second week of her hospitalization she had some psychiatric behaviors such as auditory hallucinations and she attempted suicide attempt by cutting her wrists with a sharp metal. The patient was immediately consulted to a pediatric psychiatrist. Escitalopram and risperidone treatments were started. Postpartum depression and hyponatremia (though her sodium level was $135 \mathrm{mEq} / \mathrm{L}$ at that time) was thought to be the cause of the psychiatric symptoms.

Her abdominal pain was increasing in intensity during her follow-up; paracetamol, nonsteroidal anti-inflammatory drugs and finally oral tramadol were used to relieve the pain. Toxicologic investigation for serum mercury, lead and cadmium levels were in normal range. Anti-nuclear antibody, antidsDNA and anti-neutrophil cytoplasmic antibody tests were normal. Clinical findings and laboratory investigations did not reveal any infectious etiologies.

Vomiting accompanying abdominal pain led us to stop giving anything peroral finally. Gastrointestinal endoscopy was performed to exclude gastric pathologies, it did not show any abnormalities. Oral ondansetron treatment partially decreased vomiting attacks. She did not tolerate oral nutrition; therefore total parenteral nutrition was started.

During an abdominal crisis the patient usually preferred prone position. She also had a family history of a mother dying at a young age with similar symptoms, suggesting a genetic disease. She had constipation and vomiting. The urine color of the patient was not red, but she had positive urobilinogen test in several urine analyses. Acute porphyria was the preliminary diagnosis.

The Watson-Schwartz test which is the screening test for acute porphyria was positive. To confirm the diagnosis serum and urine porphyrin levels were ordered. Urine total porphyrin level was $418 \mathrm{nmol} / \mathrm{L}(<35)$, erythrocyte protoporohyrin 
was $0.6 \mu \mathrm{mol} / \mathrm{L}(0-1.4)$, total plasma porphyrin was $26.1 \mathrm{nmol} / \mathrm{L}(0-10)$. Genetic analysis showed a heterozygous mutation in HMBS gene $(\mathrm{c} 160+6 \mathrm{~T}>\mathrm{A})$.

Intravenous fluid containing $12.5 \%$ dextrose was administered. Her abdominal pain subsided. After a few days, $3 \mathrm{mg} / \mathrm{kg} /$ day intravenous hemin was administered for two days. The patient's abdominal pain relieved. Before discharge, the patient's serum sodium was $139 \mathrm{meq} / \mathrm{L}$, creatinine was $0.97 \mathrm{mg} / \mathrm{dl}$. Her blood pressure was normal, amlodipine and enalapril treatments were discontinued. Her psychiatric symptoms subsided totally.

Informed consent was received from the family.

\section{Discussion}

Acute Intermittent Porphyria is an autosomal dominant rare metabolic disease with an estimated prevalence of one in 75.000 in Europe. ${ }^{1}$ Hydroxymethylbilane synthase (HMBS) mutations cause disorder in heme biosynthesis. Onset of symptoms may be delayed until adulthood. The most common presentation in children is abdominal pain which may mimic an acute abdomen and it may be associated with vomiting and constipation. ${ }^{3}$ Electrolyte imbalances especially hyponatremia due to SIADH are seen. Gastric and renal losses may contribute to the development of hyponatremia. Our patient was a post-partum refugee girl, who had severe abdominal pain together with SIADH induced hyponatremia.

Hypertension is a common finding in patients manifested with AIP. In a population-based retrospective study, hypertension prevalence was shown to be $56 \%$ in AIP patients and porphyrin precursors were speculated to have a cytotoxic and/or vasospastic effect on renal arteries. ${ }^{4}$ Autonomic dysfunction/ neuropathy contributes to the development of hypertension. ${ }^{5}$ Our patient was hypertensive on admission, anti-hypertensive medication was started.
Although many of the patients remain asymptomatic, the attacks of AIP might be precipitated by several factors including drugs, alcohol, low calorie intake, hormonal changes in women. ${ }^{6}$ A relation with the onset of porphyrias with birth and menstrual cycles were defined in the literature. Increased estrogen and progesterone levels may trigger acute porphyria attacks by increasing the porphyrin precursors via inducing the first enzyme in heme biosynthesis. ${ }^{7}$ In a retrospective study of Andersson et al. ${ }^{8}$, they showed that oral contraceptives caused attack in $24 \%$ of AIP patients. Our patient was healthy until the age of 17 and she had a two -year- old daughter. Her complaints started after the birth of her second child.

In a report from Belgium, a 22 -year- old female patient was admitted to the intensive care unit with severe hyponatremia and abdominal pain. Imaging of the abdomen was normal, she also had SIADH. The patient was in a premenstrual phase and she was using oral contraceptives. She was diagnosed with AIP. ${ }^{9}$

In AIP neurologic and psychiatric symptoms may be seen. The mechanism of neurological issues are not clear. However, the symptoms may result from increased neurotoxic porphyrin precursors. On admission, our patient had a seizure which probably resulted from hyponatremia. Without an interpreter's help we could not communicate with her. That caused difficulties in understanding the developing psychological issues, and finally she made a suicide attempt. She was started on escitalopram and risperidone treatment. In a case report from China, they presented a patient with SIADH who was then diagnosed with AIP, it was mentioned that the patient's mother had committed a suicide due to pain symptoms when her child was five years old. ${ }^{10}$

Singh et al. ${ }^{11}$ reported a 35 -year- old male patient, who applied to hospital with complaints of altered mental status and vomiting. The patient had severe hyponatremia due to SIADH. Despite improvement in sodium level, 
the patient had severe tonic-clonic seizures. Hemin was not available; the patient's attack was treated with hemodialysis.

A reddish color may develop in urine samples of acute porphyria patients due to the excess porphyrin precursors, especially when the urine is exposed to light. An abnormal color change was not noticed in our patient's urine, but urobilinogen was positive in her urinary analyses. The diagnosis was clinically made with the pattern of abdominal pain (she usually preferred the prone position) and togetherness of SIADH. SIADH development in acute porphyria may be associated with a vascular damage to hypothalamus due to high levels of circulating $\delta$-aminolevulinic acid (ALA) and porphobilinogen (PBG). Also, persistent nausea and vomiting may contribute to SIADH. ${ }^{9}$

High levels of ALA, PBG and porphyrin are diagnostic. Treatment consists of intravenous high dextrose fluids and hemin. Our patient's abdominal pain partially subsided with dextrose infusion. After hemin was obtained and administered, there was a remarkable improvement in her pain and mood. Genetic analysis showed a heterozygous mutation in HMBS gene (c160+6T>A).

In conclusion, acute porphyrias must be considered in differential diagnosis of abdominal pain, especially when there are accompanying symptoms like hyponatremia, seizures, mental changes and hypertension. Early diagnosis and specific treatment with hemin are important to prevent complications.

\section{REFERENCES}

1. Puy H, Gouya L, Deybach JC. Porphyrias. Lancet 2010; 375: 924-937.
2. Stein PE, Badminton MN, Rees DC. Update review of the acute porhyrias. Br J Haematol 2017; 176: 527538

3. Balwani M, Singh $\mathrm{P}$, Seth A, et al. Acute intermittent porphyria in children: a case report and review of the literature. Mol Genet Metab 2016; 119: 295-299.

4. Andersson C, Lithner F. Hypertension and renal disease in patients with acute intermittent porphyria. J Intern Med 1994; 236: 169-175.

5. Pischik E, Kauppinen R. An update of clinical management of acute intermittent porphyria. Appl Clin Genet 2015; 8: 201-214.

6. Bylesjö I, Wikberg A, Andersson C. Clinical aspects of acute intermittent porphyria in northern Sweden: a population-based study. Scand J Clin Lab Invest 2009; 69: 612-618.

7. Granick S. The induction in vitro of the synthesis of delta-aminolevulinic acid synthetase in chemical porphyria: a response to certain drugs, sex hormones, and foreign chemicals. J Biol Chem 1966; 241: 1359-1375.

8. Andersson C, Innala E, Backstrom T. Acute intermittent porphyria in women: clinical expression, use and experience of exogenous sex hormones. A population-based study in northern Sweden. J Intern Med 2003; 254: 176-183.

9. Meersseman W, Cassiman S, Goossens W, Vanderschueren S. An unusual cause of syndrome of inappropriate antidiuretic hormone secretion. Acta Clin Belg 2008; 63: 277-280.

10. Li Y, Qu H, Wang H, Deng H, Liu Z. Novel A219P mutation of hydroxymethybilane synthase identified in a Chinese woman with acute intermittent porphyria and syndrome of inappropriate antidiuretic hormone. Ann Hum Genet 2015; 79: 310-312.

11. Singh PS, Rawat R, Zafar KS. Acute intermittent porphyria with syndrome of inappropriate antidiuretic hormone secretion (SIADH) and neurological crisis, successfully treated with hemodialysis. Int J Res Med Sci 2014; 2: 795-797. 